\title{
A Low Cost Induction Motor Controller for Light Electric Vehicles in Local Areas
}

\author{
Rui Esteves Araújo*, Henrique Teixeira**, José Barbosa** and Vicente Leite ${ }^{* *}$ \\ ${ }^{*}$ Faculdade de Engenharia da Universidade do Porto, Porto, Portugal \\ ** Instituto Politécnico de Bragança, Bragança, Portugal
}

\begin{abstract}
This paper is concerned with design considerations and tradeoffs involved in the power electronics development for light electric vehicles. A review of propulsion system design, power conversion structure and control is presented. A three-phase squirrel-cage induction motor is used as propulsion system for an electric scooter. The motor is controlled at different operating conditions by means of a simple scalar control using a low cost controller board developed for light electric vehicles used in local areas. Experimental results show that the proposed digital controller is able to follow the reference speed with a suitable dynamics for the electric scooter.
\end{abstract}

\section{INTRODUCTION}

Nowadays, environmental quality of cities is an emerging issue. The ever increasing traffic volume has produced high levels of carbon dioxide emissions from the conventional gasoline and diesel-fueled vehicles, which will contribute to a substantial increase of pollution in the major cities all over the world, causing heavy consequences to the communities [1-4]. It has been recognized that electric vehicles are the only viable solution in order to reduced air pollution, in particular, in large urban areas. However, electrical vehicles have serious disadvantages because of the limitation on cruising range imposed by weight, capacity of the batteries and long recharging time. Electric scooters are a step towards the solution for the environmental problems and traffic congestion created by cars. Moreover, electric scooters are competitive when devoted to urban mobility where it is not necessary an extended driving range. Therefore, the research and development of electric scooters in order to reduce air pollution and environmental damage becomes relevant. In addition, if those disadvantages can be overcome, motorcycles customers will be motivated to purchase electric motorcycles in the near future. Therefore, our objective in this paper is to establish an appropriate platform for the study of electric motorcycles, and then to design a low cost controller for the achievements of the desired control objectives.

In this context the induction motor offers weight and efficiency advantages over the more conventional DC motors, besides its traditional advantages of robustness, low cost and well established manufacturing techniques. This work presents the design of a propulsion system for a prototype of a low cost electric scooter. The propulsion system consists of a three-phase induction motor driving the road wheels using a differential. The main benefits of these vehicles include: zero emissions, reduced noise pollution, significant reduction in maintenance and more comfortable ride from greatly reduced vibration. In this project, the viability and affordability of the electric scooter has been studied.

This present paper is organized as follows: the design methodology for an electric vehicle is reviewed in section II, section III deals with the motor drive design inside the proposed system, the experimental results are shown in section IV, and lastly the conclusions are presented.

\section{Design Methodology}

\section{A. Constraints and Specifications of the Propulsion System}

To proceed in the design of the electric motor size it is essential to determine cruising, climbing and acceleration power requirements for the propulsion systems. In particular, the main performance constraints, corresponding to different regimes, can be described by the following variables: vehicle type and mass, maximum velocity, rated speed for the specified road grade, and acceleration.

In addition, when designing a propulsion system for any battery-powered electric scooter, the main objective is to get a large charge range while achieving low drive weight, volume and cost. For this objective, the selection of the speed ratio $R$, (i.e. motor maximum speed divided by its rated speed) has a great influence on the drive efficiency, weight and volume. The speed ratio determines the range of speeds over which the motor operates in the constant torque region and the range over which it operates in constant power region. In reference [5] is shown that the peak power rating decreases as the speed ratio is increased and speed ratios above 5.0 do not lead to any substantial reduction in the peak power rating. Furthermore, high speed ratios are achieved with significant design difficulties and leads to higher fluxing conditions. This issue is important because low power rating results in lower requirements related to the power capability of the batteries and power converter.

We propose a simple analysis which permits to conclude that if a high value for the speed ratio is selected it simply increases the dimensions and weight of the motor. Suppose that we consider two induction motors with different speed ratios $R_{I}$ and $R_{2}$, respectively. The flux linkages are ideally constant up to rated speed and inversely proportional to speed in the constant power region, then the flux equations of the induction motors can be written as follows:

$$
\Phi_{1}=\frac{V}{\omega_{R_{1}}}=\frac{V}{\omega_{\max }} R_{1} \text { and } \Phi_{2}=\frac{V}{\omega_{R_{2}}}=\frac{V}{\omega_{\max }} R_{2}
$$


If $R_{1}>R_{2}$ results that $\Phi_{l}>\Phi_{2}$. In other words, the induction motor 1 will have to support a greater value of flux-turns than the motor 2. Consequently, the induction motor 1 will have a greater weight and dimensions than the induction motor 2. Evidently the speed range of the motor is limited by its pull out torque at high speed, which is proportional to the product of flux by rotor current. In the field-weakening region, flux falls with increasing frequency and the consequent reduction in torque is exacerbated by the fall in stator voltage due to effects of leakage inductance. To achieve a higher speed ratio it is necessary to minimize the leakage inductance, what it would imply to modify the design for induction motor. It was found by experimental tests for standard induction motors, allowing a safety margin, that a good speed ratio is 2 . It is important to note that for this project, a standard motor has been used without any changes in the windings layout. Furthermore, the frame size has remained unchanged.

\section{B. Review of the Vehicle Model}

A simple mathematical model is used for the vehicle. The equation of motion along the scooter's longitudinal axis is expressed by:

$$
F_{w}=f_{r}+f_{a}+f_{c}+f_{d}
$$

where $F_{w}, f_{r}, f_{a}, f_{c}$ and $f_{d}$ represent the road load, rolling resistance, aerodynamic resistance, climbing resistance and acceleration resistance, respectively.

The rolling resistance is caused by the tire deformation on the road, when the speed is lower, and can be expressed by:

$$
f_{r}=k_{r} m \mathrm{~g} \cos \theta
$$

In (3) $\theta$ represents the angle of the vehicle relatively to the horizontal, $k_{r}$ is the rolling resistance coefficient, $m$ is the vehicle mass, and $g$ is the gravitational acceleration constant.

The aerodynamic resistance, $f_{a}$, is the viscous resistance of air upon the vehicle:

$$
f_{a}=\frac{1}{2} \xi C_{w} A\left(v+v_{o}\right)^{2}
$$

$\zeta$ being the air density, $C_{w}$ is the aerodynamic drag coefficient, $A$ is the scooter frontal area with drive, $v$ is the vehicle speed and $v_{o}$ is the head wind velocity.

The climbing resistance and down grade force is given by (5), where $i$ is the slope of the road.

$$
f_{c}=m \mathrm{~g} i
$$

When the vehicle is climbing the sign is positive and when the vehicle is climbing down the sign is negative.

The acceleration resistance is related to the vehicle speed as follows:

$$
f_{d}=k_{d} m \frac{d v}{d t}
$$

where $k_{d}$ is the rotational inertia coefficient that compensates the apparent increase in the vehicle's mass due to the onboard rotating mass.

\section{Estimation of Electric Motor Power}

The first step of the propulsion system design is to estimate the power capacity of the electric motor. To do this, we need to calculate the steady-state characteristics of the electric scooter when it is climbing. The total running resistance corresponding to the specified grade is calculated and the motor minimum power is fixed equal to the vehicle's power requirement to maintain a constant speed at the specified grade:

$$
P_{\min }=\frac{v_{\text {grade }} F_{w}}{\eta}
$$

The variable $\eta$ represents the transmission efficiency.

Another important factor is the motor peak power needed to achieve a specified acceleration time or a cruising maximum speed. This determines the peak current in the power system. Thus, it establishes the current rating of the switching devices and the maximal discharge current of battery. In order to calculate the maximum power of the motor it is useful to make reference to the expression of the acceleration in terms of the net force $F-F_{w}$, which accelerates or decelerates the vehicle:

$$
a=\frac{d v}{d t}=\frac{F-F_{w}}{k_{d} m}
$$

$F$ being the traction force at the drive wheels provided by the motor. A procedure to determine the peak power rating of the drive used for the electric vehicle propulsion has been presented in [5]:

$$
P_{\max }=\frac{k_{d} m}{t_{f} \eta}\left(\frac{v_{b}^{2}+v_{f}^{2}}{2}\right)
$$

where $v_{b}$ is the vehicle speed, corresponding to the motor base speed, $v_{f}$ is the vehicle speed at the end of acceleration, and $t_{f}$ is the acceleration time. The acceleration of the vehicle from rest to a specified final speed consists of a period with rated torque, during which the motor speed increases to the base speed, followed by a period with rated power with the speed increasing until the final speed. The rated torque is taken as the value required for climbing the maximum incline. A value for the base speed must be chosen which allows the vehicle acceleration specification to be found without demanding excessive motor power.

\section{Adopted Conversion Structure}

The aim of this sub-section is to discuss the power conversion structure. For this purpose, we consider two conversion topologies: 1) a simple direct $\mathrm{DC} / \mathrm{AC}$ (voltage source inverter) and 2) a cascaded DC/DC/AC (step-up converter + voltage source inverter). In recent literature $[6,7]$ the cascaded DC/DC/AC structure has been proved to be more efficient with respect to the single inverter 
solution. However, when the overall project is oriented to cost reduction and minimization of the system complexity and when the required mechanical power is low, the second solution does not seem to be the best solution.

\section{MOTOR DRIVE DESIGN}

\section{A. Scooter Specifications}

A three-wheeled scooter derived from a 50 c.c. internal combustion engine frame has been setup. Fig. 1 shows a lateral view of the scooter. The same figure shows also the space reserved for batteries, static converters and controller, placed under the driver's seat. The scooter specifications are listed in table I. The electric scooter is supposed to have similar performance, load capacity, and autonomy of the conventional gasoline-fueled (single cylinder 2 stroke) one.

As mentioned in the section II, the motor power capacity can be determined by the load power for steadystate driving. Then the required power when driving on a climb is given by (7).

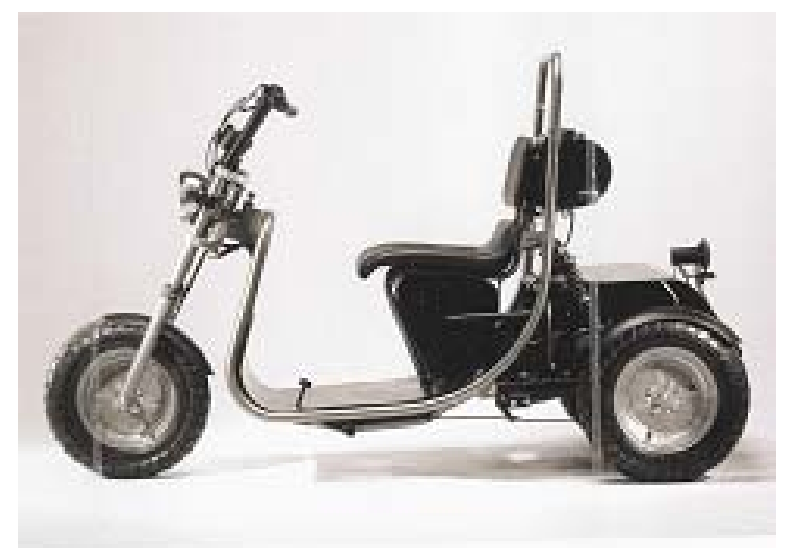

Figure 1. Framework of the three-wheeled scooter from the company Alberto Carvalho Araújo \& $\mathrm{C}^{\mathrm{a}}$., $\mathrm{L}^{\mathrm{da}}$..

TABLE I.

SCOOTER SPECIFICATIONS

\begin{tabular}{|c|c|}
\hline Length & $2026 \mathrm{~mm}$ \\
\hline Width & $930 \mathrm{~mm}$ \\
\hline Vehicle weight & $240 \mathrm{~kg}$ \\
\hline Load (one person + luggage) & $150 \mathrm{~kg}$ \\
\hline Maximum speed & $45 \mathrm{~km} / \mathrm{h}$ \\
\hline Maximum road grade at 5km/h & $20 \%$ \\
\hline 0 - 25 km/h acceleration & $10 \mathrm{~s}$ \\
\hline Driving range & $60 \mathrm{~km}$ \\
\hline Wheel radius & $486 \mathrm{~mm}$ \\
\hline Power consumed in accessories & $100 \mathrm{~W}$ \\
\hline Transmission efficiency & $92 \%$ \\
\hline
\end{tabular}

\section{B. Electric Motor Type}

In general the desired features of the motor for an electric vehicle are high torque/inertia and power/weight ratios, high speed, low maintenance, small size, low weight and cost, and high efficiency over low and high speed ranges. Permanent magnet motors and induction motors have most of the mentioned features. Permanent magnet motors have an advantage over the induction motor from the point view of operation efficiency, but these motors are more expensive and it is not easy to perform field weakening in order to increase the range of speed with such motors. Moreover, this type of machine presents recycling difficulties. This can be a big problem when these motors are used in large scale in electrical cars. On the other hand, the induction motors are less expensive, easily recyclable and requires less maintenance. For this project the motor needs a totally enclosed construction in order to protect it from external environment. The overloading possibility is limited at $30 \%$ of motor rated torque because the cooling efficiency is limited by the enclosed construction of the motor. In table II the induction motor characteristics are shown. The propulsion system consists of a single motor driving the rear wheels via a fixed ratio gearbox and differential.

\section{Power Conversion System and Control Scheme}

Fig. 2 shows the structure of the designed electric scooter. The shaft of the induction motor is directly linked with a differential gear. The electric scooter has one control unit, composed by the controller board and power inverter.

An open loop voltage/frequency control for a voltage inverter fed induction motor is used because of its simplicity and low cost. Moreover, the application requirements are completely fulfilled by this type of control. The basic principle of operation is also illustrated in fig. 2.

The speed controller board consists of a generator that produces the speed reference as well as the forward and reverse commands based on an accelerator handle. The speed reference determines the inverter frequency directly, after passing through a V/F law. At the core of the controller board is a PWM module designed to meet the demanding requirements of the induction motor drive. This module generates six center aligned PWM signals that can be connected directly to the driver circuits.

TABLE II.

INDUCTION MOTOR CHARACTERISTICS

\begin{tabular}{|c|c|}
\hline Rated power & $1.1 \mathrm{~kW}$ \\
\hline Rated torque & $7.7 \mathrm{Nm}$ \\
\hline Maximum torque & $18.6 \mathrm{Nm}$ \\
\hline Rated speed & $1400 \mathrm{rpm}$ \\
\hline Rated frequency & $50 \mathrm{~Hz}$ \\
\hline Voltage & $70 \mathrm{~V}$ \\
\hline Rated current & $16 \mathrm{~A}$ \\
\hline Weight & $11 \mathrm{~kg}$ \\
\hline
\end{tabular}




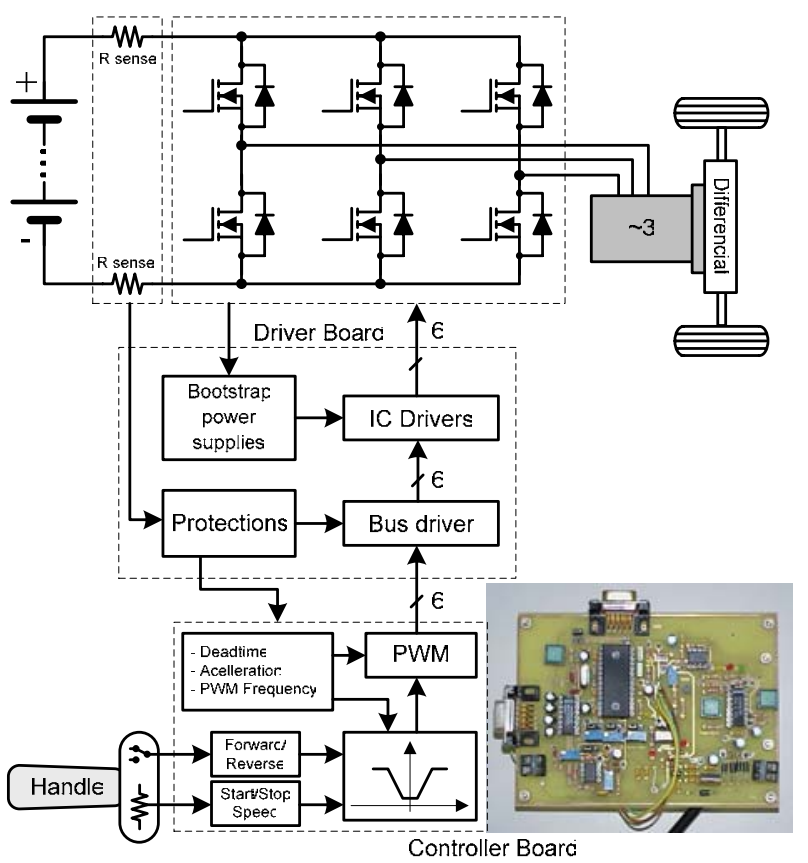

Figure 2. Block diagram of the electric scooter.

The main subsystems implemented are: generating circuit, voltage/frequency law, PWM modulator, driver circuits and power inverter. The most universally accepted motor drive topology is used, that consists of a six-switch voltage inverter driving a three-phase induction motor as shown in fig. 2 . The voltage inverter is built by means of six power MOSFETs, ref. IRFP 260N, from International Rectifier. Their drain source breakdown voltage is $200 \mathrm{~V}$, and the maximum drain current is $46 \mathrm{~A}$ (at $25^{\circ} \mathrm{C}$ ). The DC bus voltage will vary from $96 \mathrm{~V}$ to $108 \mathrm{~V}$, depending on the charge state of the batteries. A $200 \mathrm{~V}$ device would allow adequate margin above inductive $d i / d t$ voltage spikes. The MOSFETs continuous current rating is determined by power loss. Typically if a sine wave of current has a peak of $22,6 \mathrm{~A}$, the current rating of the device should be 45,2 A. Peak currents up to 46 A would be suitable so long as maximum junction temperature does not exceed $150^{\circ} \mathrm{C}$.

Others topics of circuit design that require same attention are: gate drive, physical bus structure and protection circuits. The gate drive is an important element of the inverter design. The implementation of a power supply for the high side circuitry in half-bridge inverter is problematic because the high side power supply is connected to the switching output of the half-bridge. In the driver board the bootstrap circuits are used to generate the floating power supplies for the high side circuitry of halfbridge inverters. An example of a gate drive circuit using the bootstrap technique is shown in figure 3 . These circuits provide a simple, low cost, and effective method of generating floating voltage supplies.

Considering the high $d i / d t$ of the MOSFETs, it is important to minimize stray inductance. The circuit inverter implements a low inductance DC bus by laminating together positive and negative bus bars to take advantage of flux canceling. The power board has been designed to support current protections. This is achieved by means of two shunt resistors placed on DC bus. The basic circuit was implemented as shown in fig. 4, using the high speed optocoupler HCPL2611. Fig. 5 shows the response of the protection circuit during a fault detection. The signal of channel 1 corresponds to the output of the optocoupler HCPL2611, in the circuit of fig. 4 and the signal of channel 2 corresponds to the one of the PWM inputs of the drive HCPL314J, in the circuit of fig. 3 . The respective delay is less than $200 \mathrm{~ns}$ and the overall delay since the over voltage detected in the resistances $R_{\text {sense }}$ until the gates of the MOSFETs is less than 200 ns plus the propagation delay of the HCPL2611 and HCPL314J which is less than $0.7 \mu \mathrm{s}$.

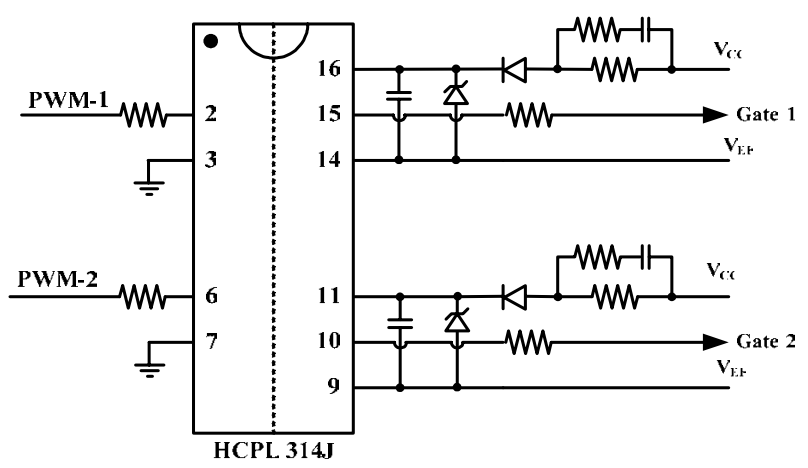

Figure 3. Electric circuit of the bootstrap power supplies for one leg of the inverter.

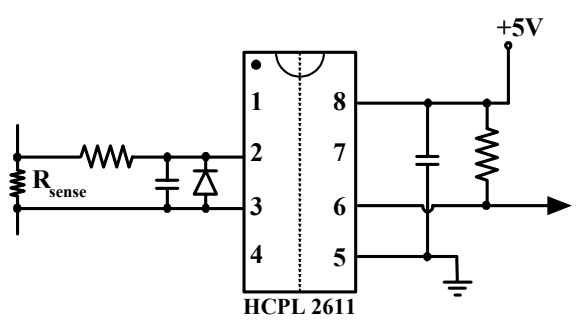

Figure 4. Electric circuit for short circuit in the positive and negative DC bus.

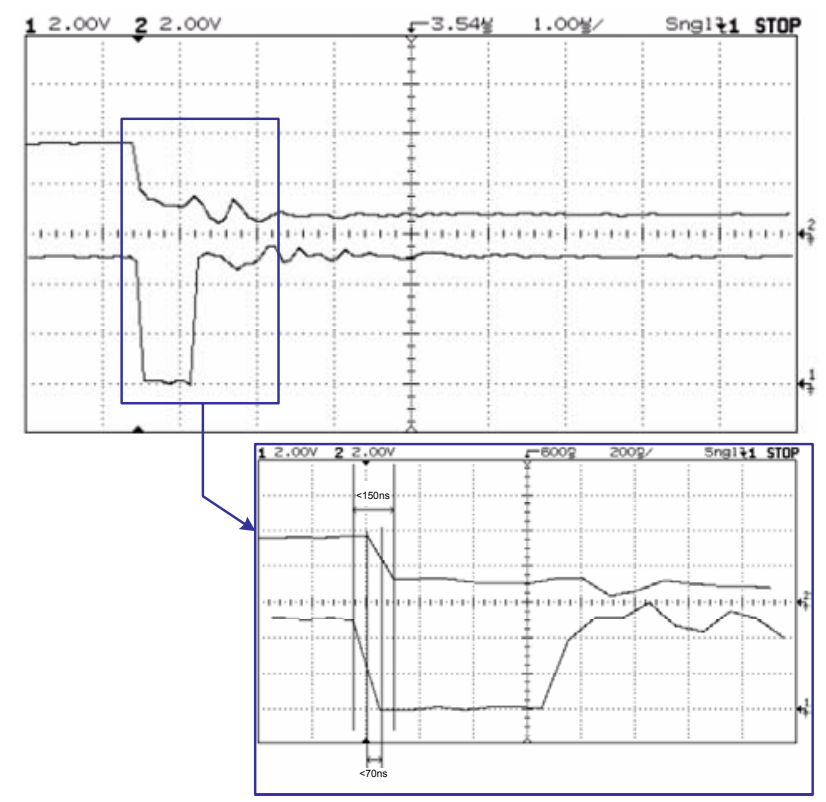

Figure 5. Response of the protection circuit during a fault detection. Channel 1 (below): Fault signal. Channel 1 (above): gate signal. 


\section{The Main Controller Board Functionalities}

The controller board is based on a microcontroller and has the general features for light electric vehicles, like Start/Stop, Forward/Reverse, and Speed commands. It enables us to set different maximum forward and reverse speeds, since the last is lower than the first one as it happens in general. The Forward/Reverse direction is only updated when the speed reference is decreased below a threshold voltage and then set again to the desired speed reference. By this way an accidental change of the vehicle direction is not possible. Even if the driver changes the direction during march he needs to decrease the speed reference below that threshold voltage and then set the new speed reference again to update the direction of the vehicle.

Although not usually available to the drivers, some others configurations can be set such as Acceleration/Deceleration time, PWM frequency, dead time and the boost at lower frequencies. In this project and by safety reasons the acceleration time is different in the forward and reverse directions.

These general configurations make the controller board suitable for use with different power structures and therefore different motor powers.

\section{E. Considerations About Different Technologies and Size of Batteries}

A light electric scooter is a typical application where the batteries are frequently charged and discharged. In such applications it is necessary to use a battery with a "deep cycle" characteristic which means that they are prepared to have many cycles of charge and discharge. Another important characteristic to have in consideration is the splashing risk of the acid that is inside the battery if the battery cracks or opens during an accident, for instance. Due to these requirements, "deep cycle", "Gelled" (or "Gel Cell") batteries can be considered as a good option since they contain acid in a form of a solid mass obtained by the addition of silica gel and therefore it is not possible to spill acid. However, this type of batteries has some disadvantages. On the one hand, they must be charged at a slower rate (typically $\mathrm{C} / 20$ ), in order to avoid excess gas from damaging the cells, and they can not be fast charged on a conventional automotive charger or they may be permanently damaged. On the other hand, they must be charged at a lower voltage than other types of batteries such as the flooded (the less expensive, but with more maintenance and less tolerant to cold) and the AGM - Absorbed Glass Mat - ones (with the higher cost). The AGM batteries have the advantages of the gelled ones, but can support worst conditions (including hot climates) without the disadvantages referred above. Therefore, this new type of sealed batteries using "absorbed glass mats" can be a better option for light electric vehicles.

In this project, eight deep cycle batteries are being used. Their main characteristics are shown in table III.

The battery size is determined by two factors; the power and the storage energy demands. The maximum continuous power is estimated considering the peak power of the electric motor. On the contrary, the storage energy is calculated considering the driving range and an estimation of the average consumption.
TABLE III.

BATTERIES CHARACTERISTICS

\begin{tabular}{|c|c|}
\hline Voltage & $12 \mathrm{~V}(\times 8)$ \\
\hline Capacity & $28 \mathrm{Ah}$ \\
\hline Maximum discharge current & $400 \mathrm{~A}$ \\
\hline N. $^{\circ}$ of cycles & $\geq 1200^{(1)}$ \\
\hline Weight & $11.7 \mathrm{~kg}$ \\
\hline Technology & "Gel" \\
\hline
\end{tabular}

${ }^{(1)}$ Discharge depth less than $60 \%$.

The battery power can be obtained from the motor mechanical power, considering the overall system efficiency $\eta_{t}$ (converter + motor), as follows:

$$
P_{b}=\frac{P_{m}}{\eta_{t}}
$$

It should be noted that some additional storage energy must be considered in order to feed accessories such as lights.

\section{EXPERIMENTAL RESULTS}

The control system of fig. 2 is implemented on the laboratory induction motor drives using a digital controller board. Extensive tests have been carried out to confirm the validity and reliability of the proposed low cost control unit.

An experimental set-up has been used for the tests in the laboratory before the ones carried out with the chassis. It consists of a test bench (with a programmable powder brake, an incremental encoder and a tachometer generator, and a torque sensor, all from Leroy Somer) and the dSPACE development system, ACE Kit 1103, based on the DS1103 PPC controller board, the Real-Time Interface (RTI) blockset for Simulink as well as experiment software (ControlDesk, MLIB/MTRACE).

\section{A. Tests with Different Speed Commands}

Fig 6(a) shows the result when a step command of about $47 \mathrm{rad} / \mathrm{s}$ is set at $6 \mathrm{~s}$, where the speed change from $103 \mathrm{rad} / \mathrm{s}$ to $150 \mathrm{rad} / \mathrm{s}$. In fig. 6(b) is shown the response of a variation in the speed reference from $105 \mathrm{rad} / \mathrm{s}$ to 150 $\mathrm{rad} / \mathrm{s}$, and then to $87 \mathrm{rad} / \mathrm{s}$. These experiments are performed in order to analyze the performance of the control unit to step variation in speed reference and also to investigate the system behavior with step increase and decrease of the speed. We can see a good speed build up and very stable performance. Fig. 6(c) shows another test with reduction in the speed reference from about $154 \mathrm{rad} / \mathrm{s}$ to $71 \mathrm{rad} / \mathrm{s}$.

\section{B. Braking mode}

Fig.7 shows the characteristics in the braking mode control. We can see in canal 2 the speed response to a change in the speed reference from $104 \mathrm{rad} / \mathrm{s}$ to $0 \mathrm{rad} / \mathrm{s}$. The canal 1 shows the stator current transient during this stop operation. 


\section{Efficiency}

Fig. 8 shows the total efficiency of the drive system including the PWM inverter and motor. The motor speed was varied from a few hundred of rpm to $2400 \mathrm{rpm}$. As can be seen the total efficiency of the drive system is higher than $60 \%$ above $1200 \mathrm{rpm}$.

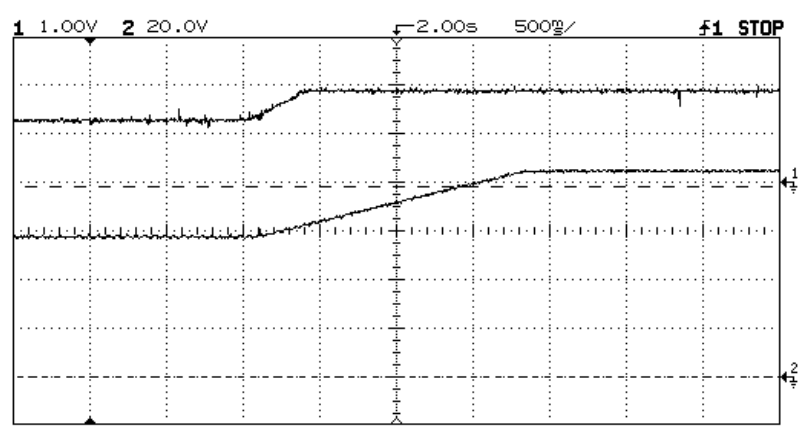

(a)

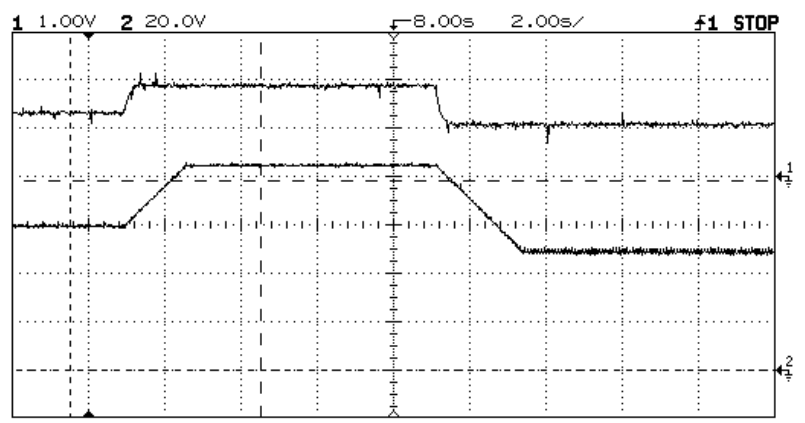

(b)

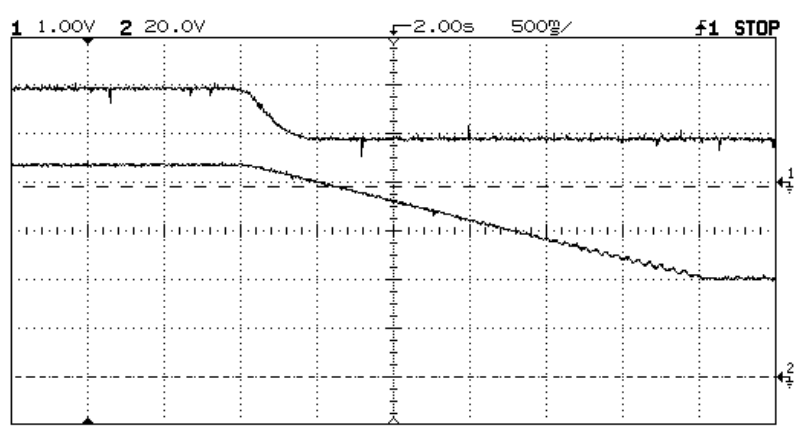

(c)

Figure 6. Tests with different speed commands.

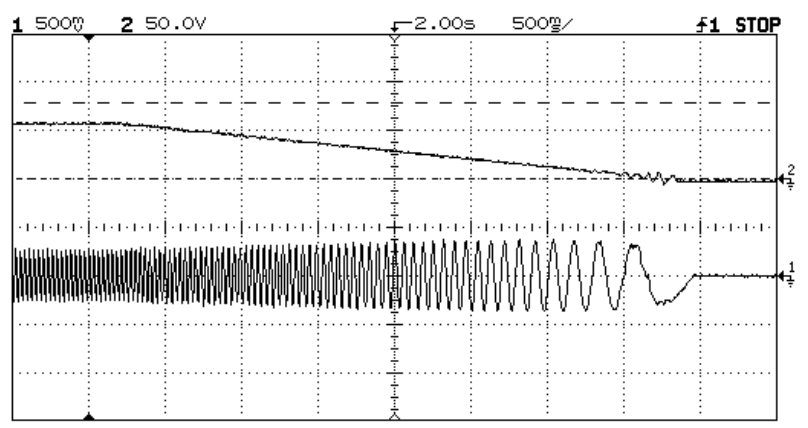

Figure 7. Braking mode.

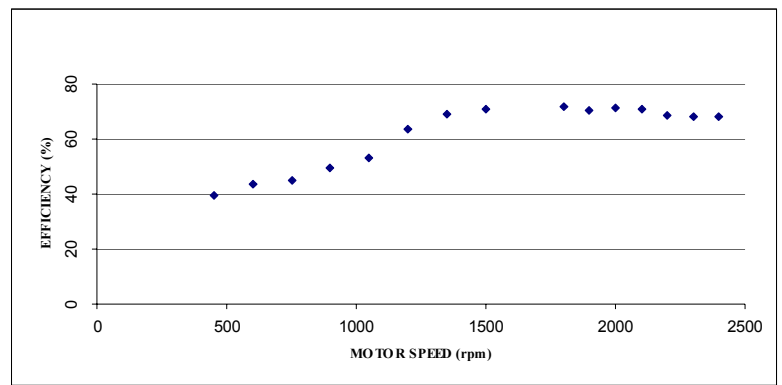

Figure 8. Total efficiency of the drive system including PWM inverter and motor.

\section{CONCLUSION}

In this paper the design of a three-wheeled electric scooter has been presented. The scalar control method is a simple and inexpensive method for induction motor control, implemented on a low cost digital controller board. The experimental results show that the implementation of the voltage/frequency control strategy on a digital platform based on a microcontroller is feasible and reliable in this kind of application concerning an electric scooter. The aim of this project was to demonstrate that light electric scooters could be a viable alternative to traditional scooters powered by internal combustion engines (two or four stroke). The prototype realization has been carried out successfully and the experimental results have shown that the controller performs a stable and efficient motor control, without penalizing performances of the conventional scooter.

\section{ACKNOWLEDGMENT}

The authors would like to acknowledge the collaboration and technical support provided by Engineer Nuno Araújo and Alberto Carvalho Araújo \& $C^{\mathrm{a}}$. $\mathrm{L}^{\mathrm{da}}$. company.

\section{REFERENCES}

[1] Y Hori, "Future Vehicle Driven by Electricity and ControlResearch on Four-Wheel-Motored UOT Electric March II", IEEE Trans. Ind. Electron., vol. 51, 954 - 962, 2004.

[2] C. Chan, "The state of the art of electric and hybrid electric", in Proceedings of the IEEE, vol. 90, $\mathrm{n}^{\circ} 2$, February, 2002.

[3] S. Naito, N. Mutoh, T. Takagi and Y. Kouchi, "AC drive systems for electric vehicles", Hitachi Review, vol. 77, n², 1995.

[4] H. Yamamura, R. Masaki, O. Koizumi, K. Naoi and S. Naito, "Development of powertrain system for Nissan FEV", in Proceedings of the $11^{\text {th }}$ Electric Vehicle Symposium, Florence, Italy, 1992.

[5] M. Ehsanim, K. M. Rahman, and H. A. Toliyat, "Propulsion system design of electric and hybrid vehicles", IEEE Trans. Ind. Electron., vol. 44, 19-27, 1997.

[6] C. Chakraborty, M.C Ta, T Hori, "Speed sensorless, efficiency optimized control of induction motor drives suitable for EV applications", in Proceedings of the $29^{\text {th }}$ Annual Conference of the Industrial Electronics Society, 2003.

[7] P. Guglielmi, G. M. Pellegrino, G. Griffero, S. Nieddu, G. Giraudo and A. Vagati, "Power conversion concepts for advanced autonomy and reliability electric scooter", in Proceedings of the $29^{\text {Th }}$ Annual Conference of the IEEE Industrial Electronics Society, November 2-6, Roanoke, Virginia, USA, 2003. 